\title{
Die Ausweitung kommunikativer Räume: Reichweite, Mechanismen und Theorien der Globalisierung der Wissenschaft ${ }^{1}$
}

\section{Von Anita Engels und Tina Ruschenburg}

\section{Die Bedeutung von Globalisierungsprozessen für nationalstaatliche Räume}

Dass unser Alltag voller Anzeichen für eine zunehmend globalisierte Welt steckt, ist kaum $\mathrm{zu}$ bestreiten. Jedoch reicht der Verweis auf preiswerte Fernreisen und das Internet nicht aus, um gesellschaftliche Transformationsprozesse hinreichend $\mathrm{zu}$ verstehen und ihre Reichweite einschätzen zu können. Besondere Aufmerksamkeit hat die sozialwissenschaftliche Globalisierungsdiskussion immer dann erzeugt, wenn sie einen direkten Zusammenhang zwischen Globalisierungsprozessen einerseits und Auflösungstendenzen des Nationalstaates oder nationaler Räume andererseits behauptet hat. So wurde in den frühen 1990er Jahren, allerdings etwas vorschnell und überwiegend ohne Rückgriff auf empirische Daten, das Ende der Volkswirtschaften bzw. das Ende des Nationalstaates ausgerufen (Ohmae 1995; Reich 1993) oder dessen Überholtheit angesichts der neuen globalen Umstände in den Vordergrund gestellt (Albrow 1996). Diese Behauptungen haben eine ganze Batterie von Gegenpositionen hervorgerufen. Insbesondere unter dem Stichwort »varieties of capitalism « wurde empirisch nachgewiesen, dass die Ökonomie nach wie vor durch nationalstaatlich geprägte Rahmenbedingungen bestimmt wird, die sich als nationale Produktionsregime oder political economies beschreiben lassen (Hall/Soskice 2001; Crouch/Streeck 1997; Schmidt 2002). Die globale Ausrichtung transnationaler Unternehmen - den Kronzeugen des behaupteten Niedergangs nationaler Räume - lasse sich nur in wenigen Fällen empirisch nachweisen, wohingegen die Mehrzahl der Unternehmen nach wie vor eine nationale Verankerung in ihrem Entstehungsland haben (Doremus et al. 1998). Auch bei Multinationalen Konzernen, die an Weltmärkten operieren, lassen sich strategische Entscheidungen der Unternehmensleitung häufig auf einen sogenannten »home country effect« zurückführen (Levy/Kolk 2002). Vorsichtigere Formulierungen in Bezug auf Denationalisierungsprozesse haben die Debatte inzwischen versachlicht (Beisheim et al. 1999). Indem grenzüberschreitende Sozialformationen an Bedeutung gewinnen, werden sie zunehmend in die alltäglichen Entscheidungsprozesse der Akteure mit einbezogen und ersetzen den nationalen Bezugsrahmen. Der Denationalisierungsthese scheinbar entgegen stehen jedoch zahlreiche Studien aus dem Bereich der neo-institutionalistischen world polity-Forschung, die gerade unter den Prämissen von Globalisierung von einer Stärkung des Prinzips der Nationalstaatlichkeit ausgehen. Indem sich Nationalstaaten weltweit als dominante Organisationsform durchsetzen und sie als legitime Akteure behandelt werden, wird Nationalstaatlichkeit als Ritual der Moderne sichtbar, das sich in den vergangenen Jahrzehnten auf immer mehr Handlungsbereiche ausgedehnt hat (Meyer 2005). Die Diskrepanz zwischen Erodierungs- und Stärkungsbehauptung lässt sich z.T. darauf zurückführen, dass die Untersuchungen auf unterschiedlichen Analyseebenen ansetzen (Guillén 2001), aber auch darauf, dass zunächst wohl beide Beobachtungen zutref-

1) Die Daten aus den Abschnitten 3, 4 und 5 stammen aus dem Forschungsprojekt »Diskurse globaler ökologischer Bedrohung. Die Globalisierung von Wissenschaft unter der Bedingung der Medialisierung «, das von der Deutschen Forschungsgemeinschaft gefördert wird (WE 972/17-2; Leitung Peter Weingart). Für wertvolle Kommentare danken wir Georg Krücken, Frank Meier, Andre Müller und einem/einer anonymen GutachterIn. John Meyer und dem Stanford Comparative Workshop danken wir für die Gelegenheit, die diesem Artikel zu Grunde liegenden Ergebnisse dort zur Diskussion zu stellen. 
fend sind - sowohl der Bedeutungsverlust von Nationalstaaten in einigen Bereichen als auch die zunehmende Verbreitung und ein steigendes Aktivitätsniveau der Nationalstaaten in anderen Bereichen (Smelser 2003).

Die sozialwissenschaftliche Globalisierungsforschung ist inzwischen in eine Phase eingetreten, in der theoretische und begriffliche Präzisierungen zwar ein differenziertes Bild erzeugen, die aber gleichzeitig auch als neue Unübersichtlichkeit bezeichnet wird (Dürrschmidt 2002, S. 7). Dabei wird Globalisierung zunehmend als umfassendes Phänomen betrachtet, das sich in verschiedene Einzeltransformationen zerlegen lässt. Transnationalisierung beschreibt beispielsweise eine Forschungsrichtung, die sich mit der Herausbildung von Sozialräumen (Pries 1996) oder Handlungsnormierungen (Djelic/Sahlin-Andersson 2006) jenseits von oder quer zu nationalstaatlichen Einflussbereichen beschäftigt. Deterritorialisierung bezieht sich auf die zunehmende Entkopplung von räumlichen und sozialen Bezügen, die vor allem im Kontext veränderter Nationalstaatlichkeit Bedeutung erlangt (Basch et al. 1994). Die Herausbildung neuer (europäischer, globaler) governance-Formen (Brock 2003; Kohler-Koch/Eising 1999), eng verbunden mit der These einer sich entwickelnden Weltöffentlichkeit oder globalen Zivilgesellschaft (Anheier et al. 2001; Beck1996), ist als weiterer Bereich zu nennen. Während frühere Arbeiten sich eher auf programmatische oder zeitdiagnostische Beiträge kapriziert haben, als empirisch fundierte Untersuchungen vorzulegen, gibt es nun eine Reihe von Beiträgen, die detailliert anhand von qualitativen Einzelstudien die Konstruktionsweisen einer sich globalisierenden sozialen Welt nachzeichnen. Nach wie vor mangelt es jedoch an großen Überblicksstudien, die Globalisierungsphänomene in verschiedenen Gesellschaftsbereichen quantitativ nachzuweisen versuchen.

Ziel dieses Artikels ist es, Globalisierungsprozesse als Ausweitung kommunikativer Räume zu untersuchen und dabei gezielt nach der Bedeutung dieser Ausweitung für den Wandel nationalstaatlicher Räume zu fragen. Dafür wählen wir das eher unübliche Beispiel der Wissenschaft. Da Wissenschaft geradezu paradigmatisch für ein bereits globales Kommunikationssystem erscheint, wird Globalisierung in diesem Bereich oft fraglos vorausgesetzt. Exemplarisch untersuchen wir dabei erstens die Reichweite des Ausweitungsprozesses. Dabei geht es um die Frage nach der faktischen Inklusion marginaler Weltregionen, die auf der Grundlage von Ungleichheits- und Abhängigkeitsmustern besonderen Bedingungen unterliegt. Systemtheoretische Ansätze zur Weltgesellschaft haben beispielsweise argumentiert, dass die fortschreitende funktionale Differenzierung alle Weltregionen erfasst und es kaum noch möglich ist, die Eigenlogik von Zahlungen (Wirtschaft) oder Wahrheitskommunikationen (Wissenschaft) staatlich zu unterbinden und anderen Systemlogiken dauerhaft unterzuordnen (Stichweh 2000). In diesem Sinne findet eine weltweite Inklusion statt. Andererseits haben gerade Vertreter der Systemtheorie argumentiert, dass durch funktionale Differenzierung regionale Differenzen derart verstärkt werden können, dass kumulative Exklusionseffekte auftreten (Luhmann 1997; Stichweh 1997). Inwiefern Inklusion unter Ungleichheits- und Abhängigkeitsbedingungen also gerade zu dem paradoxen Ergebnis der Exklusion ganzer Weltregionen führen kann, bleibt dabei offen. Zweitens suchen wir nach Evidenzen für Denationalisierungsphänomene, d.h. die abnehmende Bedeutung des nationalstaatlichen Rahmens für Austauschbeziehungen und Sozialformationen. Drittens werden wir spezifische Mechanismen herausarbeiten, die die beschriebenen Ausweitungs- (und gegebenenfalls auch die Denationalisierungs-) Prozesse vorantreiben. Die Nutzung neuer Technologien, insbesondere im Informations- und Kommunikationsbereich, wird häufig als zentraler Mechanismus genannt (Castells 1996). Wir vermuten jedoch, dass es sich dabei um eine notwendige, aber nicht hinreichende Grundbedingung für die Ausweitung kommunikativer Räume handelt. Insbesondere im Bereich der Wissenschaft werden daher zusätzliche Mechanismen vermutet, wie z.B. die fortschreitende disziplinäre Ausdifferenzierung, die zunehmende Bedeutung globaler Forschungsthemen sowie Strukturveränderungen in der Forschungsfinanzierung. 
Der folgende Abschnitt 2 stellt zunächst heraus, warum die Wissenschaft als weltweites Kommunikationssystem zur Untersuchung von Globalisierungsprozessen besonders geeignet ist. In Abschnitt 3 wird eine empirische Untersuchung vorgestellt, die sich mit der quantitativen Erfassung wissenschaftlicher Kooperationen beschäftigt. Im Vordergrund steht dabei die Entwicklung der internationalen Koautorenschaft in Publikationen von insgesamt 54 Forschungseinrichtungen in Deutschland und den USA über einen Zeitraum von zehn Jahren. In Abschnitt 4 werden ergänzend dazu die Internetauftritte dieser Forschungseinrichtungen untersucht, um deren Selbstverortungen im virtuellen kommunikativen Raum zu untersuchen. Abschnitt 5 enthält die Auswertung von Interviews, die mit den LeiterInnen der Forschungseinrichtungen sowie mit VertreterInnen deutscher und US-amerikanischer Einrichtungen zur Forschungsförderung geführt wurden. Hier werden vier Mechanismen herausgearbeitet, die in unterschiedlicher Weise zu einer Ausweitung kommunikativer Räume führen. Abschnitt 6 diskutiert abschließend die hier gefundenen Zusammenhänge von Globalisierung und nationalen Kommunikationsräumen.

\section{Globalisierung im weltweiten System der Wissenschaft?}

Wissenschaft ist wohl das gesellschaftliche Teilsystem, in der die Unterstellung eines weltweiten Kommunikationssystems am deutlichsten wirkt, nicht zuletzt durch den universalistischen Geltungsanspruch von Wahrheit. Im Unterschied zu Gesetzgebungsprozessen beispielsweise, die typischerweise eine begrenzte Gültigkeit innerhalb einer bestimmten Jurisdiktion erreichen, lässt sich die Kommunikation von Wahrheit in ihrer Reichweite nicht durch Grenzen aufhalten. Stichweh betont jedoch, dass dies nicht immer selbstverständlich mit einer globalisierten Sozialstruktur einherging, so dass »die fraglose Globalität der Wissenschaft des späten 20. Jahrhunderts « als Erklärungsproblem behandelt werden müsse (Stichweh 1999, S. 29). Vor allem disziplinäre Differenzierungen, personalisierte Netzwerke der Kooperation, die transorganisatorische (im Unterschied zur intraorganisatorischen) Nutzung von Telekommunikation, die Entstehung globaler wissenschaftlicher Probleme und räumlich unbegrenzte Netzwerkstrukturen von wissenschaftlichen Communities sind zur Erklärung der Globalisierung der Wissenschaft heranzuziehen (Stichweh 1999, S. 30f.). Hinzu kommen die weltweite Diffusion von wissenschaftlichen Einrichtungen und Hochschulsystemen, die Verankerung von Forschungsförderung als Staatsaufgabe und die Proliferation internationaler Wissenschaftsvereinigungen als Indikatoren für eine weltweite Institutionalisierung von Wissenschaft als autoritativer Stimme der Moderne (Drori et al. 2003).

Andererseits ist diese globale Möglichkeitsstruktur (die beinhaltet, dass überall auf der Welt Wissenschaft betrieben werden kann, die überall sonst wahrgenommen und zum Anlass von Vernetzungen und Kooperationen genommen werden kann) nicht gleichmäßig realisiert. Sowohl die Produktion als auch die Rezeption wissenschaftlicher Erkenntnis unterliegen faktisch einer regionalen Begrenzung. So hat Schott beispielsweise anhand von Zitationsdaten herausgestellt, dass das weltweite Kommunikationssystem der Wissenschaft in sechs verschiedene, regional verortete Kommunikationsräume differenziert ist (Schott 1988). Für Vernetzungen und Kooperationen, die im Unterschied zur bloßen Rezeption von Forschungsergebnissen meist auf face-to-face Kontakte angewiesen sind, gilt dies in verstärkter Form. Der universalistische Anspruch von Wissenschaft trifft zudem auf eine extrem ungleiche Verteilung der Teilhabechancen im Weltmaßstab (Gaillard/Krishna/Waast 1997). Sowohl der Zugang zu wissenschaftlichem Wissen als auch die Chance, an seiner Erzeugung mitzuwirken, sind in weiten Teilen der Welt äußerst limitiert. ${ }^{2}$ Zudem hat die koloniale Vergan-

2) In gewisser Hinsicht gilt sogar, dass Ungleichheit in der Wissenschaft (gemessen an der Verteilung der Weltpublikationstätigkeit) größer ist als Ungleichheit in der Wirtschaft (gemessen an der Verteilung des GNP) (Frame/Narin/Carpenter 1977; für eine ähnliche Argumentation vgl. Schott 1988, S. 225). 
genheit auch im Bereich der Wissenschaft zu Abhängigkeitsverhältnissen geführt, die im 19. und frühen 20. Jahrhundert spezifische Formen der Kolonialwissenschaft hervorgebracht haben (Raina/Habib 2004). Diese Ungleichheits- und Abhängigkeitsverhältnisse werden z.T. auch gegenwärtig als neokoloniale oder neoimperialistische Strukturen der globalen Wissenschaft interpretiert (Dahdouh-Guebas et al. 2003; Goldman 2004). Marginale Weltregionen, die nicht auf eine eigene (vorkoloniale) Wissenschaftstradition zurückblicken können, sind heute zum Teil vollständig aus dem wissenschaftlichen Kommunikationssystem exkludiert (Engels 2003). Es ist also daher eine offene Frage, wie die potentiell weltumspannenden Kommunikationsbeziehungen in einem gegebenen Feld und einem gegebenen Zeitraum jeweils unterschiedlich realisiert werden.

Außerdem hat die Wissenschaftsforschung gezeigt, dass ungeachtet des Universalitätsanspruchs die konkreten Erzeugungs- und Verwertungszusammenhänge wissenschaftlichen Wissens häufig durch nationalstaatliche Kontexte geprägt sind. Da sich Wissenschaft im 18. und 19. Jahrhundert vor allem im Kontext von Nationalstaaten herausgebildet hat, zeichneten sich bereits früh Unterschiede in nationalen Stilen der Forschung ab (Jamison 1982). Der nationale Rahmen prägt die Erzeugung von wissenschaftlichem Wissen durch die Bereitstellung von notwendigen Ressourcen und Infrastruktur und stellt gleichzeitig einen Kontext für die politische Verwertung (Politikberatung) und die ökonomische Verwertung (nationale Innovationssysteme) dieses Wissens dar (Nelson 1993; Engels/Weingart 1997). Dies bedeutet möglicherweise ein Spannungsverhältnis zwischen universalistischem Anspruch wissenschaftlicher Geltung und kontextgebundener Forschungspraxis, das sich auch in unterschiedlichen Selbstbeschreibungen widerspiegelt: einerseits als »Wissenschaft« (ohne Artikel), in deren Begriff sich der Universalismusanspruch niederschlägt, und andererseits als verschiedene regional geprägte Wissenschaften. So ist z.B. immer wieder von westlicher Wissenschaft die Rede, oder von nationaler Forschung (z.B. die deutsche Forschung in der Antarktis, die deutsche Forschung auf dem Prüfstand) und nationaler Wissenschaft (z.B. der Buchtitel »Herausforderung für die deutsche Wissenschaft«, WBGU 1996).

Vor diesem Hintergrund ist es also interessant, die »Globalität der Wissenschaft des späten 20. Jahrhunderts « nicht nur als Erklärungsproblem zu betrachten, sondern gerade die Fraglosigkeit dieser Ausgangsbeobachtung zu überprüfen. Welche Aspekte von Wissenschaft sind globalisiert, welche Aspekte sind weiterhin durch den nationalstaatlichen Kontext geprägt, und wie hängen diese Beobachtungen zusammen? Vor diesem Hintergrund werden wir nun die Prozesse der Globalisierung im Sinne einer Ausweitung kommunikativer Räume sowie der Denationalisierung auf das Wissenschaftssystem beziehen und nach möglichen Operationalisierungen dieser Fragestellungen suchen.

Globalisierung: Hierbei geht es um die Ausweitung bisher lokal begrenzter kommunikativer Räume und um die Reichweite dieses Prozesses. Dabei ist vor allem eine Verdichtung bereits bestehender Kontakte und Vernetzungen innerhalb der OECD-Welt zu erwarten. Eine Ausweitung auch auf marginale Entwicklungsländer wäre in dem Sinne von Bedeutung, dass sie angesichts der extremen Ungleichheit im Weltmaßstab voraussetzungsvoll und unwahrscheinlich ist und daher einen besonders harten Indikator für Globalisierung im hier verstandenen Sinne darstellt.

Denationalisierung: Um Denationalisierung beobachten zu können, muss man zunächst davon ausgehen, dass eine nationale Einbettung von Wissenschaft (Erzeugungs- und Verwertungskontexte, organisatorische Ausstattung) gegeben ist. Denationalisierung würde immer dann beobachtet werden, wenn die Ausweitung kommunikativer Räume mit abnehmender kommunikativer Dichte innerhalb des eigenen nationalen Kontexts einhergeht, wenn also eine Verschiebung vom nationalen zum übernationalen oder globalen Kontext zu beobachten ist. Ein wichtiges Anzeichen dafür wäre beispielsweise ein Schrumpfen nationaler 
Forschungsförderung, oder ein Rückgang von Kooperationen mit inländischen Partnern (vgl. Crawford et al. 1993). Auch ein nachlassendes Interesse an nationalen Wissenschaftsgesellschaften, deren Publikationen und Konferenzen wäre ein interessanter Hinweis.

Ein zentraler Zugang der Wissenschaftsforschung zur Erfassung kommunikativer Räume ist die Nutzung von Publikationsdatenbanken wie z.B. dem Science Citation Index. Hier kann z.B. anhand der angegebenen Autorenadressen nachvollzogen werden, in welchem Umfang bestimmte Personen, wissenschaftliche Einrichtungen oder Nationen gemeinsam publizieren. So kann die Frage nach einer Ausweitung von Koautorenschaftsbeziehungen relativ problemlos auch für große (fachlich oder regional abgegrenzte) Untersuchungseinheiten beantwortet werden. Hierzu hat sich unter dem Begriff Bibliometrie ein eigenes Forschungsfeld ausdifferenziert (für einen Überblick vgl. Luukkonen et al. 1992).

Wir werden im folgenden drei verschiedene Datenquellen nutzen, um zumindest einige der hier angesprochenen Fragen an einem empirischen Fallbeispiel testen zu können. Zu einem ausgewählten Sample von deutschen und US-amerikanischen Forschungseinrichtungen analysieren wir erstens Koautorenschaftsmuster der Publikationen, die aus diesen Instituten hervorgegangen sind. Dabei wird getrennt nach Anzeichen für Globalisierung und Denationalisierung im oben beschriebenen Sinne gefragt. Die zweite Datenquelle besteht aus den Internetdarstellungen der ausgewählten Forschungseinrichtungen, um die Analyse des kognitiven Kerns der Wissenschaft (Fachpublikationen) um die Untersuchung der Verortung der dazugehörigen organisatorischen Einheiten im virtuellen kommunikativen Raum zu ergänzen. Als dritte Datenquelle nutzen wir Interviews, die mit VertreterInnen US-amerikanischer und deutscher Forschungs- und Forschungsförderungs-Einrichtungen geführt wurden, um Hinweise auf Mechanismen für die Ausweitung kommunikativer Räume zu finden.

Um einen möglichst aussagekräftigen Fall zu untersuchen, haben wir Institute und InterviewpartnerInnen aus dem Bereich »globale Umweltforschung « ausgewählt, in dem deutlich sichtbare Globalisierungsphänomene zu erwarten sind. Globale Umweltforschung bezieht sich auf die Erfassung und Bewertung der ökologischen Selbstgefährdung der Gesellschaft im planetaren Maßstab. Es geht darum, Wissen über kritische ökologische Zustände zu systematisieren und gesellschaftliche Folgen globaler Veränderungen sichtbar zu machen. Sie zielt auf die Analyseeinheit der Erdsysteme und der globalen Dynamiken ab und ist deshalb in besonderer Weise darauf angewiesen, Daten und Beobachtungen aus allen Teilen der Welt in einen gemeinsamen Analyserahmen zu integrieren, um zu allgemein gültigen Aussagen zu kommen. Gleichzeitig geht es um die Begründung von umfangreichen Handlungsprogrammen für die Nutzung von knappen Ressourcen und gegen die Verschmutzung von Ökosystemen. Globale Umweltforschung ist dabei immer im Kontext von internationalen Umweltverhandlungen zu sehen, die das Ziel möglichst weltumfassender Schutzabkommen verfolgen. ${ }^{3}$ Sowohl kognitiv als auch organisatorisch ist die globale Umweltforschung daher auf die Globalisierung ihrer eigenen Grundlagen ausgerichtet. Der Vergleich von deutschen und USamerikanischen Forschungseinrichtungen ist insofern interessant, als der nationale Kontext hierdurch variiert werden kann. Allerdings stellen Deutschland und die USA dabei trotz aller Unterschiede hinreichend ähnliche nationale Kontexte dar, da es sich in beiden Fällen um Kontexte mit einer ausgebildeten Forschungsinfrastruktur, hohen Forschungsausgaben und einer ausgeprägten Forschungspolitik handelt. Gerade im Hinblick auf Globalisierungsprozesse ist der Fall der USA jedoch besonders interessant: Zum einen haben viele Prozesse, die sich in den USA abspielen, aufgrund der schieren Größe des Landes weltweite Ausstrah-

3) Die bedeutendsten sind wohl das Montreal-Abkommen zum Schutz der stratosphärischen Ozonschicht, die Klimarahmenkonvention mit dem darauf aufbauenden Kyoto-Protokoll sowie die Biodiversitätskonvention, die dem Verlust der Artenvielfalt entgegen zu wirken beabsichtigt. 
lungseffekte. Zum anderen wird den USA in vielen Bereichen eine isolationistische Grundhaltung unterstellt, die sich in der Wissenschaft als nach innen gewandte Ausrichtung der Forschungskooperationen äußern könnte.

\section{Die bibliometrische Untersuchung der Entwicklung von Koautorenschaft}

Wir nutzen die Publikationsdaten aus den Datenbanken des Web of Science ${ }^{4}$ um herauszufinden, welche nationalen und übernationalen Koautorenschaftsbeziehungen es in der deutschen und US-amerikanischen Global-Change-Forschung gibt und wie sie sich im Laufe einer Dekade verändert haben. Gemeinschaftliche Veröffentlichungen haben eine lange Tradition, die bis zum Beginn der Professionalisierung der Wissenschaft im 17. Jahrhundert zurückreicht (Beaver/Rosen 1978). Obgleich diese Form der Kooperation also kein rezentes Phänomen ist, zeigen neuere Studien, dass sich in den vergangenen Jahrzehnten ein genereller Trend zu internationaler Koautorenschaft abzeichnet, der jedoch erheblich mit Disziplinen und Nationen variiert (Leclerc/Gagné 1994, Glänzel 2001). Je größer das Publikationsvolumen eines Landes ist, desto geringer ist in der Regel der Anteil von Artikeln mit Koautoren aus anderen Ländern. Dies wird meist damit erklärt, dass es für spezialisierte Wissenschaftler in großen Ländern leichter ist, Experten auf dem gleichen Gebiet im eigenen Land zu finden, als für Forscher in kleinen Ländern. Gleichzeitig besteht in kleinen Ländern häufig ein stärkerer Druck, Geräte und Ressourcen international zu teilen (Luukkonen et al. 1992, Schubert/Braun 1990).

Grundlage unserer bibliometrischen Untersuchung sind die Publikationen der 54 ausgewählten Institute in den Jahren 1993-2002. Um die Eigenschaften der deutschen und der USamerikanischen Global-Change-Forschung vergleichen zu können, wurde für beide Länder jeweils ein Sample aus den Publikationen aller Institute gebildet. Das deutsche Sample umfasst insgesamt 10.087 Publikationen von 18 Forschungseinrichtungen, das US-amerikanische enthält 37.111 Artikel aus 36 Instituten. Die jährliche Zahl von Publikationen ist im Verlauf des untersuchten Zeitraums bei beiden Samples deutlich angestiegen. Alle in diesen Artikeln angegebenen Adressen wurden ausgewertet. Im folgenden wird erstens die grundlegende Frage nach einer Ausweitung der Kooperationsbeziehungen sowie der Reichweite dieses Prozesses untersucht; zweitens wird nach Evidenzen für eine Denationalisierung der Kooperationsbeziehungen gesucht. ${ }^{5}$

\section{Die Entwicklung grenzüberschreitender Koautorenschaft als Indikator für Globalisie- rung}

Um zu prüfen, ob zwischen 1993 und 2002 eine Zunahme grenzüberschreitender Forschungskooperationen zu beobachten ist, wurde der jährliche Anteil der Artikel mit internationaler Koautorenschaft berechnet (vgl. Tab. 1). ${ }^{6}$ Artikel mit internationaler Koautorenschaft werden im folgenden als internationale Artikel bezeichnet.

4) Science Citation Index, Social Science Citation Index und Arts and Humanities Citation Index.

5) Für eine ausführliche Darstellung der Methode und der Ergebnisse vgl. Engels/Ruschenburg/Weingart (2005). Eine Aufstellung aller untersuchten Forschungseinrichtungen findet sich in Anhang I.

6) Die absoluten Artikelzahlen, die diesen Anteilswerten zugrundeliegen, sind im Anhang II, Tabelle 7 zu finden. 
Tabelle 1: Anteil der Artikel mit internationaler Koautorenschaft

\begin{tabular}{|l|c|c|c|c|c|c|c|c|c|c|}
\hline & 1993 & 1994 & 1995 & 1996 & 1997 & 1998 & 1999 & 2000 & 2001 & 2002 \\
\hline $\begin{array}{l}\text { Deutsche } \\
\text { Institute }\end{array}$ & $26,9 \%$ & $31,8 \%$ & $34,2 \%$ & $34,6 \%$ & $40,4 \%$ & $40,3 \%$ & $44,0 \%$ & $49,6 \%$ & $48,7 \%$ & $50,7 \%$ \\
\hline US-Institute & $19,8 \%$ & $22,2 \%$ & $23,1 \%$ & $26,2 \%$ & $28,8 \%$ & $32,4 \%$ & $33,3 \%$ & $34,3 \%$ & $36,3 \%$ & $37,6 \%$ \\
\hline
\end{tabular}

Bei den deutschen Instituten steigt der Anteil der internationalen Artikel fast kontinuierlich von 26,9\% im Jahr 1993 auf 50,7\% im Jahr 2002, bei den US-Instituten erhöht er sich im gleichen Zeitraum von 19,8\% auf 37,6\%. Für beide Ländersamples verdoppelt sich dieser Wert also annähernd. Allerdings liegt er in dem deutschen Sample deutlich höher als in dem US-amerikanischen. Dieser Unterschied bleibt über den gesamten Untersuchungszeitraum erhalten, was mit dem vielfach beschriebenen Effekt konsistent ist, dass große Länder im allgemeinen eine geringere Tendenz zu internationalen Kopublikationen aufweisen als kleinere (s.o.). ${ }^{7}$ Die Ergebnisse zeigen, dass sowohl in der deutschen als auch in der US-amerikanischen Forschung zu globalen Umweltveränderungen in dem untersuchten Jahrzehnt ein starker Trend zu internationalen Kopublikationen herrscht. Dies stimmt mit Befunden überein, dass es in den letzten Jahrzehnten im allgemeinen ein Anstieg von Veröffentlichungen mit internationaler Koautorenschaft zu beobachten war (vgl. z.B. Leclerc/Gagné 1994, National Science Board 2004, S. 5-40). Im Vergleich zu anderen Disziplinen ist allerdings das Internationalitätsniveau der Forschung zu globalen Umweltveränderungen überdurchschnittlich hoch (Engels, Ruschenburg, Weingart 2005, S. 80).

Die sich anschließende Frage lautet, ob mit dieser Intensivierung grenzüberschreitender Kooperationsbeziehungen auch eine geographische Ausweitung verbunden ist. Hier stellt sich also die Frage nach der Reichweite des Globalisierungsprozesses: Bildet sich ein vollständig weltumspannender Kommunikationszusammenhang heraus, und falls nicht, welche Regionen sind mit einbezogen, welche nicht? Zur Beantwortung dieser Fragen wurden zunächst alle in den Koautorenadressen vorkommenden Adressen nach der UN-Klassifikation in Industrieländer, Entwicklungsländer und Übergangsländer (d.h. die früheren Ostblockstaaten) kodiert. Dies ermöglicht es, den allgemeinen Internationalisierungsindikator weiter zu differenzieren und zu prüfen, ob einzelne Länderkategorien überproportional an der Intensivierung grenzüberschreitender Kooperationen beteiligt sind (Tab. 2). ${ }^{8}$

7) Aus diesem Grund wird gelegentlich argumentiert, es sei am sinnvollsten, US-Daten mit EU-Daten vergleichen, um Publikationssysteme mit ähnlichem Umfang aneinander zu messen und so den Landesgrößeneffekt zu reduzieren. Um zu prüfen, ob die oben gefundene Differenz tatsächlich eher auf den Größenunterschied als auf nationale Spezifika zurückgeht, wurde der Anteil von internationalen Artikeln für das deutsche Sample erneut berechnet, wobei nur diejenigen Publikationen als international gezählt wurden, die mindestens eine Koautorenadresse außerhalb der EU aufweisen. In der Tat nivelliert sich der Unterschied zwischen den beiden Samples in diesem Vergleichsmodus fast vollständig (Engels/Ruschenburg/Weingart 2005, S. 73f.).

8) Dazu wird jeweils der Anteil der Artikel, deren Koautorenadressen mindestens ein Land aus der jeweiligen Länderkategorie enthalten, an allen internationalen Artikeln berechnet. Da eine Publikation auch Adressen aus mehreren Länderkategorien enthalten kann, addieren sich die Werte zu mehr als hundert Prozent auf. Die absoluten Artikelzahlen, die diesen Anteilswerten zugrundeliegen, sind im Anhang II, Tabelle $8 \mathrm{zu}$ finden. 
Tabelle 2: Anteile der Artikel mit Koautorenadressen in anderen Industrieländern, Entwicklungsländern und Übergangsländern an allen Artikeln mit internationaler Koautorenschaft

\begin{tabular}{|c|c|c|c|c|c|c|c|c|c|c|}
\hline & 1993 & 1994 & 1995 & 1996 & 1997 & 1998 & 1999 & 2000 & 2001 & 2002 \\
\hline \multicolumn{11}{|c|}{ Deutsche Institute } \\
\hline $\begin{array}{l}\text { Industrie- } \\
\text { länder }\end{array}$ & $74,2 \%$ & $79,7 \%$ & $77,2 \%$ & $77,9 \%$ & $80,8 \%$ & $78,4 \%$ & $78,8 \%$ & $80,4 \%$ & $80,3 \%$ & $75,8 \%$ \\
\hline $\begin{array}{l}\text { Entwick- } \\
\text { lungsländer }\end{array}$ & $17,0 \%$ & $17,1 \%$ & $12,8 \%$ & $14,5 \%$ & $13,8 \%$ & $18,7 \%$ & $12,7 \%$ & $15,9 \%$ & $14,5 \%$ & $17,8 \%$ \\
\hline $\begin{array}{l}\text { Übergangs- } \\
\text { länder }\end{array}$ & $15,7 \%$ & $13,8 \%$ & $19,0 \%$ & $19,8 \%$ & $17,6 \%$ & $17,8 \%$ & $18,2 \%$ & $16,3 \%$ & $15,3 \%$ & $18,8 \%$ \\
\hline \multicolumn{11}{|l|}{ US-Institute } \\
\hline $\begin{array}{l}\text { Industrie- } \\
\text { länder }\end{array}$ & $85,9 \%$ & $85,3 \%$ & $86,3 \%$ & $84,6 \%$ & $84,8 \%$ & $84,9 \%$ & $83,5 \%$ & $84,6 \%$ & $82,7 \%$ & $81,8 \%$ \\
\hline $\begin{array}{l}\text { Entwick- } \\
\text { lungsländer }\end{array}$ & $13,7 \%$ & $16,5 \%$ & $16,0 \%$ & $18,1 \%$ & $17,4 \%$ & $18,5 \%$ & $19,5 \%$ & $19,5 \%$ & $21,5 \%$ & $22,9 \%$ \\
\hline $\begin{array}{l}\text { Übergangs- } \\
\text { länder }\end{array}$ & $6,4 \%$ & $5,3 \%$ & $5,2 \%$ & $5,5 \%$ & $6,5 \%$ & $6,4 \%$ & $7,1 \%$ & $7,0 \%$ & $6,9 \%$ & $6,2 \%$ \\
\hline
\end{tabular}

Ein relativ gleich bleibender Anteil bedeutet in dieser Darstellung, dass die Koautorenschaftsbeziehungen der jeweiligen Länderkategorie an dem allgemeinen Trend zur Intensivierung grenzüberschreitender Publikationen teilhaben, sich aber nicht überproportional entwickeln. Vor diesem Hintergrund ist die Entwicklung der internationalen Publikationen der US-Institute allerdings auffällig, bei der die Entwicklungsländerbeteiligung zwischen 1993 und 2002 kontinuierlich von 13,7\% auf 22,8\% steigt, d.h. wesentlich stärker als der durchschnittliche Trend. Dies ist zunächst ein deutlicher Indikator für eine Ausweitung der Kooperationsbeziehungen über den bereits verdichteten OECD-Raum hinweg. Interessant ist nun die Differenzierung der Entwicklungsländer nach Weltregionen (vgl. Tab. 3). Hier zeigt sich eine Diskrepanz zwischen Ostasien und Mittel- und Südamerika auf der einen Seite und den übrigen Teilen Asiens, dem Großteil Afrikas sowie Ozeanien auf der anderen Seite. Die Kooperationsbeziehungen mit der ersten Gruppe von Ländern nehmen im gesamten Untersuchungszeitraum einen substantiellen Stellenwert unter allen Artikeln mit internationaler Koautorenschaft ein. Die deutschen Institute veröffentlichten 3,2\% aller internationalen Papiere mit Einrichtungen in Ostasien sowie 5,2\% mit Einrichtungen in Südamerika. Bei den US-Instituten entfielen 5,7\% der Publikationen mit internationalen Koautoren auf Ostasien und 5,0\% auf Südamerika sowie 2,4\% auf Mittelamerika. Gebiete wie ganz Afrika mit Ausnahme der Region südliches Afrika, die Karibik, Ozeanien sowie außerdem im deutschen Sample Mittelamerika, im US-Sample West- und Südostasien sind nur vereinzelt in den untersuchten Veröffentlichungen vertreten (jeweils weniger als 1\%). 
Tabelle 3: Anteil der Artikel mit Koautorenadressen in Entwicklungsländern an allen internationalen Artikeln, differenziert nach Weltregionen?

\begin{tabular}{|c|c|c|c|c|c|c|}
\hline & \multicolumn{3}{|c|}{ Deutsche Institute } & \multicolumn{3}{|c|}{ US-Institute } \\
\hline & 1993-1997 & 1998-2002 & 1993-2002 & 1993-1997 & 1998-2002 & 1993-2002 \\
\hline \multicolumn{7}{|l|}{ Afrika } \\
\hline $\begin{array}{l}\text { Östliches } \\
\text { Afrika }\end{array}$ & $0,6 \%$ & $0,6 \%$ & $0,6 \%$ & $0,6 \%$ & $0,9 \%$ & $0,8 \%$ \\
\hline $\begin{array}{l}\text { Nördliches } \\
\text { Afrika }\end{array}$ & $0,5 \%$ & $0,4 \%$ & $0,4 \%$ & $0,2 \%$ & $0,1 \%$ & $0,2 \%$ \\
\hline $\begin{array}{l}\text { Südliches } \\
\text { Afrika }\end{array}$ & $0,9 \%$ & $1,2 \%$ & $1,1 \%$ & $2,0 \%$ & $1,5 \%$ & $1,6 \%$ \\
\hline $\begin{array}{l}\text { Westliches } \\
\text { Afrika }\end{array}$ & $0,6 \%$ & $0,6 \%$ & $0,6 \%$ & $0,1 \%$ & $0,1 \%$ & $0,1 \%$ \\
\hline Zentralafrika & $0,0 \%$ & $0,1 \%$ & $0,1 \%$ & $0,2 \%$ & $0,1 \%$ & $0,1 \%$ \\
\hline \multicolumn{7}{|l|}{ Asien } \\
\hline Ostasien & $2,5 \%$ & $3,5 \%$ & $3,2 \%$ & $4,5 \%$ & $6,4 \%$ & $5,7 \%$ \\
\hline $\begin{array}{l}\text { Südzentral- } \\
\text { asien }\end{array}$ & $1,3 \%$ & $1,9 \%$ & $1,7 \%$ & $2,1 \%$ & $1,9 \%$ & $2,0 \%$ \\
\hline Südostasien & $1,3 \%$ & $1,9 \%$ & $1,7 \%$ & $0,8 \%$ & $0,9 \%$ & $0,8 \%$ \\
\hline Westasien & $1,6 \%$ & $1,0 \%$ & $1,2 \%$ & $0,5 \%$ & $0,4 \%$ & $0,4 \%$ \\
\hline \multicolumn{7}{|l|}{ Amerika } \\
\hline Mittelamerika & $0,8 \%$ & $0,4 \%$ & $0,5 \%$ & $1,5 \%$ & $2,9 \%$ & $2,4 \%$ \\
\hline Karibik & $0,4 \%$ & $0,5 \%$ & $0,5 \%$ & $0,1 \%$ & $0,7 \%$ & $0,5 \%$ \\
\hline Südamerika & $5,0 \%$ & $5,3 \%$ & $5,2 \%$ & $4,3 \%$ & $5,4 \%$ & $5,0 \%$ \\
\hline \multicolumn{7}{|l|}{ Ozeanien } \\
\hline Melanesien & $0,1 \%$ & $0,0 \%$ & $0,0 \%$ & $0,6 \%$ & $0,2 \%$ & $0,3 \%$ \\
\hline Mikronesien & $0,1 \%$ & $0,0 \%$ & $0,0 \%$ & $0,2 \%$ & $0,1 \%$ & $0,2 \%$ \\
\hline Polynesien & $0,0 \%$ & $0,0 \%$ & $0,0 \%$ & $0,1 \%$ & $0,0 \%$ & $0,1 \%$ \\
\hline
\end{tabular}

Entsprechend geht der deutliche Trend zur Ausweitung der Kooperationsbeziehungen mit Entwicklungsländern in der US-amerikanischen globalen Umweltforschung überwiegend auf eine überproportionale Intensivierung der Zusammenarbeit mit den ohnehin stark vertretenen Regionen zurück. Unter internationalen Artikeln der US-Institute, die im Untersuchungszeitraum sowohl relativ als auch absolut zugenommen haben, stellten Einrichtungen in Ostasien mit Staaten wie China einschließlich Taiwan, Hongkong und Südkorea in 4,5\% aller internationalen Artikel zwischen 1993 und 1997 und sogar von 6,4\% zwischen 1998 und 2002 die Koautoren. Die geographisch naheliegenden Länder in Mittel- und Südamerika bauen ihren von Beginn an hohen Stellenwert ebenfalls aus: Mit südamerikanischen Koautoren wurden in den ersten fünf Jahren des Untersuchungszeitraums 4,3\%, in den zweiten fünf Jahren 5,4\% aller internationalen Artikel veröffentlicht. Auch in Mittelamerika stieg der Anteil erheblich von $1,5 \%$ auf $2,9 \%$, ebenso in der Karibik von $0,1 \%$ auf $0,7 \%$. Bei den

9) Die absoluten Zahlen, auf deren Grundlage diese Prozentwerte berechnet wurden, sind in Anhang II, Tabelle 9 nachzulesen. 
Publikationen der deutschen Forschungseinrichtungen zeigt sich eine überproportionale Steigerung der Koautorenschaft neben Südamerika (von 5,0\% 1993-1997 auf 5,3\% 1998-2002) und Ostasien (von 2,5\% auf 3,5\%) auch in Südost- und Südzentralasien (jeweils von 1,3\% auf $1,9 \%)$.

Sowohl in dem deutschen als auch in dem US-Sample handelt es sich bei den stärksten regionalen Schwerpunkten Ostasien und Südamerika um Wissenschaftscommunities, die auf einem wesentlich höheren Niveau arbeiten können als die der meisten anderen asiatischen und afrikanischen Länder. Im starken Kontrast dazu bleiben die von Beginn an marginalen wissenschaftlichen Communities in Afrika, den Inselstaaten und Teilen Asiens über den gesamten Untersuchungszeitraum hinweg weitestgehend exkludiert. ${ }^{10}$ Anzeichen für den Aufbau einer stabilen Kooperation finden sich hier nicht, vielmehr erscheint die gemeinsame Veröffentlichungspraxis hier häufig als diskontinuierlich. Als Ausnahmen könnten sich bei Fortsetzung des Trends jedoch die Koautorenschaftsbeziehungen der US-Institute mit der Karibik und Ostafrika sowie die der deutschen Einrichtungen mit Südost- und Südzentralasien erweisen. Die bisherige Entwicklung lässt sich dagegen eher im Sinne einer ausgeweiteten Triadisierung als im Sinne eines durchgesetzten globalen Kommunikationsraums interpretieren.

\section{Die Entwicklung inländischer Koautorenschaft als Indikator für Denationalisierung}

Die Frage, ob Kooperationen innerhalb des eigenen Landes angesichts des festgestellten Ausweitungstrends an Bedeutung verlieren, lässt sich bibliometrisch untersuchen, indem der Anteil von Publikationen mit ausschließlich inländischen Koautoren an allen Publikationen eines Jahrgangs berechnet wird (vgl. Tab. 4). ${ }^{11}$

Tabelle 4: Anteil der Artikel mit ausschließlich inländischer Koautorenschaft

\begin{tabular}{|l|c|c|c|c|c|c|c|c|c|c|}
\hline & $\mathbf{1 9 9 3}$ & $\mathbf{1 9 9 4}$ & $\mathbf{1 9 9 5}$ & $\mathbf{1 9 9 6}$ & $\mathbf{1 9 9 7}$ & $\mathbf{1 9 9 8}$ & $\mathbf{1 9 9 9}$ & $\mathbf{2 0 0 0}$ & $\mathbf{2 0 0 1}$ & $\mathbf{2 0 0 2}$ \\
\hline $\begin{array}{l}\text { Deutsche } \\
\text { Institute }\end{array}$ & $21,8 \%$ & $21,3 \%$ & $22,7 \%$ & $20,9 \%$ & $23,1 \%$ & $24,1 \%$ & $23,8 \%$ & $21,6 \%$ & $22,8 \%$ & $23,4 \%$ \\
\hline US-Institute & $43,0 \%$ & $42,3 \%$ & $43,0 \%$ & $42,9 \%$ & $41,6 \%$ & $43,5 \%$ & $44,0 \%$ & $43,7 \%$ & $43,8 \%$ & $42,4 \%$ \\
\hline
\end{tabular}

Bei den deutschen Instituten liegt der Anteil der inländischen Artikel im Untersuchungszeitraum zwischen $20,9 \%$ und $24,1 \%$, bei den US-Instituten zwischen $41,6 \%$ und $44,0 \%$. Erneut besteht insgesamt eine deutliche Differenz zwischen den deutschen und den US-amerikanischen Werten. Wiederum ist sie zum Teil aus dem Ländergrößeneffekt zu erklären. Anders als beim Anteil internationaler Publikationen lässt sich jedoch bei keinem der beiden Samples ein Aufwärts- oder Abwärtstrend erkennen. Im Gegenteil erscheinen die Werte für

10) Vgl. auch Paraje/Sadana/Karam (2005) in einer Studie zur Entwicklung von Koautorenschaftsbeziehungen im Bereich Gesundheitsforschung zwischen 1992 und 2001, also einem durchaus vergleichbaren Untersuchungszeitraum. Die Autoren zeigen, dass der selektive Ausweitungsprozess die gleichen Ländergruppen einbezieht wie im Bereich globaler Umweltforschung: Korea, China und insbesondere amerikanische Schwellenländer erleben einen >Aufstieg «, während weite Teile Afrikas südlich der Sahara noch einmal geschwächt werden. Den Hinweis darauf verdanken wir einem/einer anonymen GutachterIn.

11) Dabei haben wir als inländische Kopublikationen alle Artikel gewertet, die mindestens eine Adresse außerhalb des erhobenen Forschungsinstituts, aber keine internationalen Koautorenadressen aufweisen. Eine dritte, komplementäre Kategorie bilden alle übrigen Artikel, die keine anderen Adressen als die institutseigene enthalten (vgl. Tab. 5). Die absoluten Artikelzahlen, die diesen beiden Tabellen zugrundeliegen, sind im Anhang II, Tabelle $7 \mathrm{zu}$ finden. 
beide Gruppen bemerkenswert konstant. Wir fassen dies als klaren Hinweis darauf auf, dass der beobachtete Trend zu mehr internationalen Kopublikationen nicht zuungunsten der nationalen Kooperationen stattfindet.

Vielmehr ist ausschließlich der Anteil der Publikationen ohne Koautoren außerhalb des eigenen Instituts rückläufig (vgl. Tab. 5). Dies gilt sowohl für das deutsche als auch das USamerikanische Sample. Kooperation im allgemeinen spielt folglich bei Publikationen in dem untersuchten Jahrzehnt eine zunehmend wichtige Rolle. Während die Zusammenarbeit mit internationalen Koautoren stark zunimmt, behalten inländische Kopublikationen ihren Stellenwert. Die bibliometrischen Daten lassen also keinen Prozess der Denationalisierung erkennen.

Tabelle 5: Anteil der Artikel ohne Koautoren außerhalb des jeweiligen Instituts

\begin{tabular}{|l|c|c|c|c|c|c|c|c|c|c|}
\hline & $\mathbf{1 9 9 3}$ & $\mathbf{1 9 9 4}$ & $\mathbf{1 9 9 5}$ & $\mathbf{1 9 9 6}$ & $\mathbf{1 9 9 7}$ & $\mathbf{1 9 9 8}$ & $\mathbf{1 9 9 9}$ & $\mathbf{2 0 0 0}$ & $\mathbf{2 0 0 1}$ & $\mathbf{2 0 0 2}$ \\
\hline $\begin{array}{l}\text { Deutsche } \\
\text { Institute }\end{array}$ & $51,3 \%$ & $46,9 \%$ & $43,0 \%$ & $44,5 \%$ & $36,5 \%$ & $35,6 \%$ & $32,2 \%$ & $28,8 \%$ & $28,5 \%$ & $25,8 \%$ \\
\hline US-Institute & $37,2 \%$ & $35,5 \%$ & $33,9 \%$ & $30,8 \%$ & $29,6 \%$ & $24,1 \%$ & $22,7 \%$ & $22,0 \%$ & $19,9 \%$ & $20,1 \%$ \\
\hline
\end{tabular}

Lesen wir die Ergebnisse der bibliometrischen Analyse, um etwas über einen Strukturwandel in der deutschen und US-amerikanischen globalen Umweltforschung zu erfahren, ergibt sich zusammenfassend folgendes Bild: Es lässt sich im Untersuchungszeitraum die Ausweitung des kommunikativen Raumes Wissenschaft beobachten, hier operationalisiert als Kopublikationen, die ein Resultat von Kooperationsbeziehungen sind. Diese Ausweitung kann als Globalisierungsprozess interpretiert werden, der deutlich über die bisherige OECDWelt hinausgeht, dabei jedoch nicht gleichmäßig in alle Weltregionen hineinstrahlt. Der Globalisierungsprozess trifft offenbar auf markante Ungleichheitsbedingungen, die verhindern, dass marginale Wissenschafts-Communities in den Ausweitungsprozess inkludiert werden. Gleichzeitig behält der nationale Kommunikationsraum aber offenbar seine Bedeutung; in gewissem Sinne wird er sogar dadurch gestärkt, dass immer weniger Publikationen von einzelnen Autoren veröffentlicht werden. D.h. insgesamt wird Koautorenschaft bedeutender, was zu einer gleich bleibenden Dichte des nationalen Kommunikationsraumes beiträgt.

\section{Die Internetauftritte als kommunikative Selbstverortung im virtuellen Raum}

Eine zweite Frage lautet, inwiefern eine globalisierte Weltsicht Eingang gefunden hat in das Selbstverständnis und die Selbstbeschreibungen der Forschungsinstitute, und welche Rolle die Einbettung der Institute in den nationalen Bedeutungsraum spielt. Hierzu untersuchen wir die Internetauftritte der Institute. Seit der flächendeckenden Einführung des Internet in den 1990er Jahren haben sich seine Nutzungsmöglichkeiten vervielfältigt. Für Organisationen im Allgemeinen wie auch für die hier untersuchten Forschungseinrichtungen im Besonderen ist die Selbstdarstellung im Internet inzwischen zu einer wichtigen Aufgabe geworden. Die Einrichtung der Homepage ist häufig bereits weit vorangetrieben, wenn ein neugegründetes Institut die tatsächliche Arbeit erst aufnimmt. Ein Forschungsinstitut, das keine Homepage vorweisen kann, gilt vielen inzwischen als suspekt, und die Abwesenheit einer solchen Selbstdarstellungsform lässt Zweifel an der Existenz der Organisation als solcher aufkommen. Dass das Internet als Ort der strategischen Selbstdarstellung gar nicht hoch genug eingeschätzt werden kann, bezeugt auch die unübersichtlich gewordene Flut an Beratungsliteratur, die sich mit der Suche nach der optimalen Homepage-Gestaltung beschäftigt. Die Internetseiten müssen die Erwartungen verschiedener Publikumssegmente erfüllen: Geberorganisationen, andere (konkurrierende) Institute, Wissenschaftler (u.a. als potentielle Mit- 
arbeiter oder als potentielle Teilnehmer einer vom Institut durchgeführten Tagung) sowie interessierte »Laien « und Massenmedien.

Der hier dargestellte Untersuchungsschritt fragt also danach, inwiefern sich Globalisierung als Wert oder Zielvorstellung in diesen Internetauftritten wiederfindet und welche Rolle die Verortung im nationalen Forschungs- und forschungspolitischen Kontext spielt. Dabei gilt es zu beachten, dass im Unterschied zur bibliometrischen Analyse keine Entwicklung, sondern immer nur eine Momentaufnahme untersucht werden kann. Die hier zugrunde liegende Momentaufnahme wurde im Dezember 2002 erhoben, markiert damit also das Ende des Untersuchungszeitraums der bibliometrischen Analyse.

Jede der 54 Forschungseinrichtungen in unserem Sample verfügt über einen eigenen Internetauftritt, und in allen Fällen ist darin zumindest in minimaler Weise eine Aussage veröffentlicht, in der sich das Institut eine Identität gibt und sich selbst im Verhältnis zur übrigen Welt beschreibt. Da die Internetauftritte zum Teil sehr umfangreich sind, war eine Auswahl notwendig: Es wurde derjenige Teil gewählt, der sich explizit der Selbstbeschreibung des gesamten Instituts, seiner spezifischen Ausrichtung und seiner Ziele und Aufgabenstellung widmet. Auf den englischsprachigen Seiten entsprach dies meist dem Abschnitt, der mit dem Begriff »mission statement « überschrieben war. So wurden 54 unterschiedlich lange Textabschnitte ausgewählt, die mit Hilfe des Programms Atlas.TI ausgewertet wurden. Die Codierung erfolgt an solchen Textstellen, die einen gesellschaftlichen Bezug herstellen, der über die kognitiven Elemente von Forschung, also etwa der bloßen Darstellung von Forschungsthemen, hinausgeht. Das ist z.B. der Fall, wenn ein Verweis auf Forschungskooperationen oder Bezüge zu anderen Instituten erfolgt oder auf eine bestimmte (forschungs-)politische Funktion, die das Institut übernimmt. Diese Textpassagen wurden jeweils als national oder global codiert.

Zunächst ist auffällig, wie vielfältig die Möglichkeiten sind, die von den Forschungseinrichtungen genutzt werden, um sich über den nationalen Bedeutungsrahmen hinaus zu positionieren. Dabei ist der Verweis auf grenzüberschreitende Kooperationen nur die naheliegendste Möglichkeit. Es finden sich jedoch auch zahlreiche Verweise auf einen selbstdefinierten Auftrag, der auf einen globalen Kommunikationsraum ausgerichtet ist:

»Connected to an international network of collaborators, SERC trains future generations of scientists to address ecological questions of the nation and the globe.« (SERC)

»It is the goal of the Institution to be a world leader in advancing and communicating a basic understanding of the oceans and their decisive role in addressing global questions.« (WHOI)

»Mit diesem Forschungsansatz leisten die Mitarbeiter des Museums Koenig einen wichtigen Beitrag zum Schutz der biologischen Ressourcen der Erde, denn die Forschungsergebnisse werden der scientific community weltweit zur Verfügung gestellt.« (ZFMK)

Zudem finden sich zahlreiche Verweise auf die grenzüberschreitende Mobilität im Kontext der Forschungstätigkeiten:

$»(\ldots)$ our research often takes us to field sites in Africa, Asia and parts of North, Central and South America.«(EEBP)

»Lamont scientists observe the earth, from its deepest interior to the outer reaches of the atmosphere, on every continent and every ocean, providing the scientific basis for the difficult choices faced by humankind in its stewardship of our planet.« (LDEO)

Aufschlussreich sind auch die Identitätskonstruktionen, die durch die von den Instituten getroffenen Vergleiche und Selbstpositionierungen nach außen kommuniziert werden. In einigen Fällen finden sich explizit Verweise auf globale Vergleichshorizonte. 
»Driven by this urgent imperative, Missouri Botanical Garden scientists conduct the most active and geographically widespread botanical research program in the world.« (MBG)

Insgesamt zeigt sich jedoch, dass auch der nationale Bezugsrahmen in den Internetauftritten der untersuchten Institute einen festen Platz einnimmt. Häufig geschieht dies mit dem Verweis auf einen nationalen Auftrag:

»What does the Pacific Marine Environmental Laboratory do for the nation? (...) PMEL currently leads the National Tsunami Hazard Mitigation Program. Payoffs: Tsunami evacuation maps are now available in the five west coast states and the real-time deep ocean detectors are providing more accurate forecasts of warnings to reduce false alarms. « (PMEL)

$»(\ldots)$ to provide and ensure timely access to global environmental data from satellites and other sources to promote, protect, and enhance the Nation's economy, security, environment, and quality of life.« (NGDC)

»To assure that our nation maintains leadership in this endeavor, we are committed to excellence in scientific investigation, in the development and operation of space systems and in the advancement of essential technologies. «(GSFC)

Deutsche Institute wählen zumeist weniger patriotische Untertöne, stellen aber ebenso häufig eine nationale Aufgabenstellung heraus:

»Mitarbeit und leitende Funktionen in zahlreichen nationalen Fachgremien« (FAL)

»Das Institut will einen eigenen Beitrag zur Verankerung von Forschung und Wissenschaft in der Gesellschaft leisten, zur Qualitätssicherung des deutschen Forschungssystems beitragen, nationale, europäische und internationale Vernetzung der Wissenschaft weiter intensivieren, verstärkt Koordinierungsaufgaben übernehmen und durch neue Forschungsergebnisse und Prognosen Entscheidungsgrundlagen für Gesellschaft, Politik und Wirtschaft zu verantwortlichem und nachhaltigem Handeln schaffen.« (MPI-Met)

»Schwerpunkte: Vernetzung der Botanischen Gärten im deutschsprachigen Raum um diese organisatorisch zu stärken, zu ihrer internen Meinungsfindung beizutragen und deren Bedeutung für Öffentlichkeit und Entscheidungsträger klarer herauszustellen.«(BIBG)

»Mit der Gründung einer zentralen Service-Einrichtung für die Bedürfnisse der deutschen Klimaforschung wurde der wachsenden Bedeutung der von der Klimaforschung zu beantwortenden Fragen nach der zukünftigen Entwicklung des Erdklimas und der sich daraus ergebenden Konsequenzen für die Umwelt des Menschen Rechnung getragen.« (DKRZ)

Auch in den Selbstpositionierungen ist der nationale Vergleichsrahmen nach wie vor von Bedeutung:

»Das IFU ist damit eines der ältesten auf dem Gebiet der atmosphärischen Umweltforschung tätigen Institute in Deutschland.« (IFU)

»Das Herbarium enthält insgesamt 1,4 Millionen Belege. Hamburg besitzt damit die viertgrößte Sammlung Deutschlands.«(IABBG)

»Das Zoologische Forschungsinstitut und Museum Alexander Koenig (ZFMK) ist eines der großen naturgeschichtlichen Forschungsmuseen in Deutschland.« (ZFMK)

»Located on a 16-acre campus on Virginia Key in Miami, it is the only subtropical applied and basic marine and atmospheric research institute of its kind in the continental United States. (RSMAS)

Besonders aufschlussreich sind Beispiele, in denen ein nationaler Bezug durch die Einbindung in einen globalen Rahmen gleichsam transzendiert wird:

»It contains the world's largest collection of Wisconsin plants.«(WSH/DBH)

Diese Auswahl von Beispielzitaten demonstriert, dass die Internetauftritte der Forschungsinstitute geeignet sind, die hier vorgeschlagenen Fragestellungen auch auf die Selbstbeschreibungen an zentralen Orten der Wissenschaft zu beziehen. Allerdings konnte bei 7 von 54 Instituten keine Selbstbeschreibung festgestellt werden, die über die bloße Benennung der Forschungsthemen hinausging, so dass sich die weitere Analyse auf 47 Institute bezieht. Im 
Anschluss an die Codierung einzelner Textausschnitte wurde für jedes dieser Institute ein Profil erstellt, d.h. es wurde bestimmt, ob es sich überwiegend um eine nationale Selbstverortung handelt (ganz ohne oder mit einigen globalen Bezügen) oder um eine globale Selbstverortung (mit einigen oder ganz ohne nationale Bezüge). Erstellt man diese Institutsprofile, so kommt man zu folgendem Ergebnis (Tab. 6). Lediglich acht Institute weisen sich als überwiegend global verortete Institute aus, wohingegen 39 von 47 Instituten eine überwiegend nationale Selbstverortung in ihrer Internetdarstellung aufweisen. Dabei ist zu beachten, dass es keinerlei signifikanten Zusammenhang zwischen den Daten für Koautorenschaftsmuster und den hier erstellten Institutsprofilen gibt. Es finden sich sowohl Institute mit einem hohem Anteil internationaler Artikel, die in ihrer Internetdarstellung eine überwiegend nationale Selbstverortung vornehmen als auch umgekehrt; bei wieder anderen Instituten findet sich ein ähnlicher Grad an Globalität in beiden Datenquellen.

Tabelle 6: Selbstverortungen der Institute in ihren Internetauftritten

\begin{tabular}{|l|c|c|c|}
\cline { 2 - 4 } \multicolumn{1}{c|}{} & US & D & ges. \\
\hline Nationale Selbstverortung (ohne globale Bezüge) & 9 & 5 & 14 \\
\hline Nationale Selbstverortung (mit einigen globalen Bezügen) & 15 & 10 & 25 \\
\hline Globale Selbstverortung (mit einigen nationalen Bezügen) & 3 & 1 & 4 \\
\hline Globale Selbstverortung (ohne nationale Bezüge) & 3 & 1 & 4 \\
\hline Gesamt & $\mathbf{3 0}$ & $\mathbf{1 7}$ & $\mathbf{4 7}$ \\
\hline
\end{tabular}

Wir interpretieren die Ergebnisse dahingehend, dass die nationale Einbettung von Forschung nach wie vor zentral ist und dass sich nur wenige Institute in ihrer Selbstverortung ganz davon lösen. Globalität spielt also nur eine untergeordnete Rolle in den Selbstbeschreibungen der Forschungseinrichtungen. Es ist zudem interessant zu sehen, dass sich die Befunde für die amerikanischen und die deutschen Institute sehr ähneln.

Globale Selbstverortungen beziehen sich dabei nur teilweise auf die Darstellung von Forschungskooperationen im engeren Sinne. Häufig stellen die Institute heraus, dass sie Daten (oder Dienstleistungen) für andere Nutzer bereitstellen, ohne dass dies zu einer Zusammenarbeit führen muss bzw. oft auch ohne dass die Nutzer bekannt sind oder durch die Inanspruchnahme des Dienstes bekannt werden. Eine andere Form der weltweiten Selbstverortung besteht darin, dass das Institut selbst »überall auf der Welt« Forschungstätigkeiten entfaltet, ohne dabei auf Kooperationen $\mathrm{zu}$ verweisen. Dem Forschungsthema »globale Umweltveränderungen« scheint außerdem inhärent zu sein, dass unabhängig von einer wissenschaftlichen Selbstverortung im engeren Sinne auch »globale Verantwortung « übernommen wird, z.B. für verbesserte Umweltbedingungen, die Erhaltung der Artenvielfalt oder den Schutz des globalen Klimas. In diesem Zusammenhang werden auch Beiträge des jeweiligen Instituts zu den internationalen Umweltverhandlungen genannt. Diese Muster lassen sich gleichermaßen in deutschen und US-amerikanischen Instituten nachweisen. Allerdings zeigt sich ein gravierender Unterschied: In acht von 47 Instituten wird der Welthorizont über die Figur der »global leadership« oder »world leadership« hergestellt, die für das eigene Institut reklamiert wird. Dabei handelt es sich ausschließlich um amerikanische Institute, wobei diese Beobachtung auch für Institute gilt, die zunächst als nicht besonders groß oder führend in einem umfassenderen Sinne auffallen. Die Bereitschaft amerikanischer Institute, sich in einem bestimmten Feld als die weltweit Besten zu stilisieren, scheint generell vorhanden zu sein und wird in schwierigen Fällen über die Definition eines entsprechend kleinen Bereiches gerechtfertigt, z.B. in dem Verweis auf »the world's largest collection in Wisconsin plants« (WSH/DBH). Hier zeigt sich, dass die Verfügbarkeit dieses Selbstbeschreibungs- 
musters offenbar national bedingt ist; im US-amerikanischen Kontext gilt das als normal, im deutschen Kontext kommt es nicht vor. ${ }^{12}$

Insgesamt zeigt diese Untersuchung, dass der diskursive Raum, in dem sich die Forschungsinstitute bewegen, erstaunlich wenig zur Demonstration der eigenen Globalität genutzt wird. Die Relevanz nationaler Räume ist auch im globalen Kommunikationssystem der Wissenschaft ungebrochen. ${ }^{13}$ Dies gilt offenbar auch in einem Forschungsgebiet, in dem es um die Erzeugung einer planetaren Perspektive geht und das deshalb besonders dazu prädestiniert scheint, national begrenzte kommunikative Räume zu transzendieren. Da es sich um Forschungseinrichtungen handelt, die zunächst einmal den organisatorischen und infrastrukturellen Rahmen für die Durchführung von Forschungstätigkeiten bereitstellen, sind sowohl die Verortungen im nationalen als auch die im ausgeweiteten Kommunikationsraum als Reaktion auf legitimatorische Erfordernisse zu interpretieren. Im folgenden Abschnitt wird anhand von Interviewmaterial untersucht werden, inwiefern sich die legitimatorischen Rahmenbedingungen in der vergangenen Dekade verändert haben, um den Befund aus der Analyse der Internetauftritte besser deuten zu können.

\section{Globalisierung der Wissenschaft in der Wahrnehmung von Forschungs- und Forschungsfördereinrichtungen}

Als dritte Datenquelle ziehen wir nun Interviews hinzu, die 2003 mit Leiterinnen und Leitern ausgewählter Forschungseinrichtungen geführt wurden, ${ }^{14}$ sowie 2004 mit den für globale Umweltforschung zuständigen Personen in der Deutschen Forschungsgemeinschaft, im Bundesministerium für Bildung und Forschung, im Deutschen Zentrum für Luft- und Raumfahrt und im Wissenschaftlichen Beirat Globale Umweltveränderungen auf der deutschen Seite sowie in der National Science Foundation, den National Academies, der National Atmospheric and Space Administration und der National Oceanic \& Atmospheric Administration auf amerikanischer Seite. Dabei haben wir gezielt nach der Bedeutung von nationalen und internationalen Kooperationen gefragt, nach Veränderungen von Kooperationsmustern im Laufe des vergangenen Jahrzehnts, sowie nach den Anreizstrukturen und den Barrieren für grenzüberschreitende Kooperationen im Bereich globaler Umweltforschung, sowohl in Bezug auf den OECD-Raum als auch darüber hinaus. Die Interviews ergeben ein umfassendes Bild über den hier untersuchten Globalisierungsprozess, da sie sowohl aus der Perspektive der Institute als auch aus der Sicht der jeweiligen nationalen Forschungsförderorganisationen Einschätzungen wiedergeben.

In zahlreichen Interviews wird darauf verwiesen, dass die (auch selbst beobachtete) Globalisierung wissenschaftlicher Kooperationsbeziehungen erst durch die kommunikationsund informationstechnischen Veränderungen der letzten Jahre möglich geworden ist. Das betrifft sowohl den erleichterten Zugang zu Informationen als auch die erheblich vereinfachte Kommunikation zwischen potentiellen Kooperationspartnern - Austausch beinahe in Echtzeit wird von den meisten ForscherInnen als inzwischen durchgesetzter Standard beschrieben. Allein die verbesserten Möglichkeiten zur Kooperation werden allerdings nicht als hinreichende Erklärung gesehen. Der zentrale Anreiz für die tatsächliche Anbahnung von

12) Interviews belegen zudem, dass dies bewusst so gewählt wird - auch von deutschen Instituten, die sich sehr wohl als Weltspitze in einem bestimmten Gebiet verstehen, wird dies auf der Internetseite nicht so benannt, weil das in Deutschland als unschicklich gilt.

13) Dies gilt zumindest für die Mehrheit der Institute. Ob die kleine Gruppe von Instituten, für die dies nicht gilt, eine wachsende Population ist, ob sich also zukünftig eine diskursive Entwicklung hin zu mehr Globalität beobachten lässt, kann mit dieser Momentaufnahme nicht geklärt werden.

14) Es wurden Interviews mit den Leiterinnen und Leitern von 39 Forschungseinrichtungen aus dem Sample geführt. 
(nationalen wie internationalen) Kooperationen ist ein erhöhter Druck von Seiten der Forschungsfördereinrichtungen, Ressourcen nach Effizienzkriterien einzusetzen. Großgeräte müssen zunehmend mit anderen Forschungseinrichtungen geteilt werden, das gilt im hier untersuchten Bereich vor allem für Forschungsschiffe, Satelliten und Großrechner. Datenbestände müssen für andere Einrichtungen aufbereitet und zur Nutzung angeboten werden. Eine verbesserte Abstimmung der Förderorganisationen untereinander verringert zudem das Duplizieren von Forschungsanstrengungen. In den USA betrifft das insbesondere die lange Zeit parallel geführten militärischen und zivilen Satellitenprogramme. Insgesamt findet ein verstärkter Wettbewerb um die zur Verfügung stehenden Ressourcen statt, bei der die Kooperation mit anderen Partnern die Chancen auf Mittelzuweisung erhöht. Zudem ist gerade für die Erforschung globaler Umweltveränderungen die Zusammenarbeit mit Forschungseinrichtungen aus möglichst vielen Weltregionen notwendig, um Zugang zu Daten, Material und generell zum Feld zu erhalten. In der Selbstdarstellung vieler InstitutsleiterInnen ist die Notwendigkeit zur weltweiten Kooperation inzwischen quasi naturalisiert: Man findet immer wieder die Aussage, dass es gar nicht anders gehe, als globale Umweltveränderungen auch unter globaler Beteiligung zu erforschen - Forschungsglobalisierung wird als ein dem Gegenstand angemessener und geradezu alternativloser Vorgang beschrieben.

Die Ergebnisse der Abschnitte 3 und 4 haben gezeigt, dass dieser Vorgang regional sehr unterschiedlich zum Tragen kommt, also eine geographisch differenzierte Reichweite entwickelt, und dass offenbar trotzdem nationale Kooperationen ihre Bedeutung beibehalten. Die Analyse der Interviews ergibt wiederkehrende Muster, aus denen sich insgesamt vier unterschiedliche Mechanismen herausarbeiten lassen:

\section{- Wissenschaftlicher Wettbewerb und strategische Partnerschaft unter Gleichen}

Viele Forschungskooperationen kommen durch individuelle Begegnungen zustande, die aus der direkten Forschungstätigkeit heraus motiviert sind. Der erhöhte Wettbewerbsdruck führt jedoch bei vielen Forschungseinrichtungen dazu, auch über die individuelle Ebene hinaus strategisch nach Kooperationspartnern auf gleichem oder höherem wissenschaftlichen Niveau zu suchen, um dadurch die Chancen auf Mittelzuweisung zu erhöhen. Die Suche nach solchen Partnerschaften erstreckt sich zunehmend über die nationalen Grenzen hinaus. Das gilt auch für US-amerikanische Forschungseinrichtungen, die lange Zeit in vielen Bereichen als weltweit führend galten und daher kaum hoffen konnten, durch grenzüberschreitende Partnerschaften ein verbessertes wissenschaftliches Niveau zu erreichen. In vielen Interviews wurde aber geäußert, dass inzwischen einzelne kanadische oder europäische Forschungsinstitute als gleichrangig gelten. Insgesamt führt dieser Mechanismus zu einer Verdichtung der Kooperationen zwischen weltweit führenden Forschungseinrichtungen in bestimmten Gebieten. Der große Anteil der internationalen Publikationen mit anderen OECD-Staaten, der sich in unserer bibliometrischen Analyse zeigt, könnte mit diesem Mechanismus eng zusammenhängen.

\section{- Politisch geförderte regionale Verdichtungen}

Ein zweiter Mechanismus lässt sich am ehesten als politische Anreizstruktur beschreiben. Das bezieht sich auf den Versuch von Forschungsfördereinrichtungen, bestimmte geographische oder politische Räume als kommunikativ verdichtete Wissenschaftsräume zu stärken. Das augenscheinlichste Beispiel für diesen Mechanismus ist die EU-Forschungsförderung, die von vielen deutschen InstitutsleiterInnen als zunehmend bedeutsame Motivation für die Anbahnung von grenzüberschreitenden Kooperationsformen genannt wurde. In ähnlicher Weise gibt es jedoch in den USA spezialisierte Förderprogramme für den Ausbau der Kooperationen innerhalb des gesamten amerikanischen Kontinents, also eine Ausweitung der Kooperationen mit Süd- und Mittelamerika. Ebenfalls gibt es spezifische Anreizstrukturen, den gesamten pazifischen Raum, d.h. die USA mit allen Pazifik-Anrainerstaaten, als Kommunikationsraum zu stärken. Für diese politisch motivierten För- 
derprogramme werden unterschiedlichste Begründungen genannt: zum einen politisch erwünschte Beziehungen zwischen Nachbarstaaten, aber auch eine verstärkte Reflexion wachsender wirtschaftlicher Verflechtungen, ebenso wie die Bedeutung dieser Regionen als geophysikalisch zusammenhängende Räume, die gerade im Themenbereich globaler Umweltveränderungen als Analyseeinheit sinnvoll erscheinen. Auch diese Form der Ausweitung wissenschaftlicher Kommunikationsräume stimmt mit den Ergebnissen der bibliometrischen Analyse überein.

\section{- Capacity building für marginale Wissenschafts-Communities}

Der dritte Mechanismus hängt unmittelbar mit dem Kontext der internationalen Umweltverhandlungen zusammen und bezieht sich auf die Ausweitung der Kooperationsbeziehungen auf marginale Entwicklungsländer. Programme für capacity building werden darin folgendermaßen eingebettet: Erstens spielt die Erstellung wissenschaftlicher Bestandsaufnahmen (global assessments) eine wichtige Rolle für die Verhandlungen und die Ausgestaltung der Konventionen. ${ }^{15}$ Zweitens sehen die internationalen Konventionen eine auf Dauer gestellte Beobachtung des Zustands der Erde vor (global monitoring). Drittens hat sich im Verhandlungsverlauf die Vorstellung durchgesetzt, dass global assessments und global monitoring nicht von einzelnen zentralen Wissenschaftseinrichtungen durchgeführt werden, sondern dass sich daran wissenschaftliche Einrichtungen weltweit beteiligen sollen. Viertens ist von Entwicklungsländern durchgesetzt worden, dass dazu Programme zum Aufbau von Forschungskapazitäten in Entwicklungsländern erforderlich sind. Fünftens schließlich ist nicht nur die globale Beschreibung des Zustandes der Erde erforderlich, sondern die in den Umweltabkommen berücksichtigten Prozesse sollen auch jeweils im nationalen Kontext der Unterzeichnerstaaten erfasst werden, d.h. z.B., dass jeder der 189 Unterzeichnerstaaten der Klimarahmenkonvention verpflichtet ist, in regelmäßigen Abständen Forschungsdokumente über den nationalen Ist-Zustand zu erstellen. Dafür wiederum sind standardisierte Methoden erforderlich. Die Kapazitätsprobleme in vielen Entwicklungsländern bei der Erstellung solcher Dokumente sollen durch internationale Kooperation überwunden werden. Diese Form der Kooperation ist zwar politisch wichtig zur Legitimation des gesamten Verhandlungsprozesses, aber sie schlägt sich typischerweise nicht in den Publikationen des wissenschaftlichen Mainstream nieder. Es handelt sich um Kooperationen unter Ungleichen, die von vielen westlichen Wissenschaftszentren zwar als politisch notwendig akzeptiert wird, die aber nicht als gleichwertige Wissenschaft betrachtet wird. Dies würde den Befund der bibliometrischen Analyse plausibilisieren, dass trotz der umfangreichen Programme zum capacity building die meisten Länder Afrikas und Teile Asiens nicht in die Ausweitung der kommunikativen Räume der Wissenschaft einbezogen sind.

- Durch nationale Verfügungsrechte erzwungene Kooperationen mit Schwellenländern Der vierte Mechanismus schließlich steht ebenfalls in engem Zusammenhang mit den internationalen Konventionen, insbesondere mit der Biodiversitätskonvention, wirkt sich jedoch völlig anders auf Kooperationsmuster aus. Forschungsthematisch interessant im Bereich der Biodiversität sind die artenreichen Gebiete der Erde, die überwiegend im Regenwaldgürtel der Erde und somit in Entwicklungsländern liegen. Bis vor wenigen Jahren galt es bei vielen Forschungsaktivitäten der Wissenschaftszentren als unproblematisch, in die entsprechenden Länder einzureisen, ohne Konsultationen mit einheimischen Forschungsein-

15) Das Intergovernmental Panel on Climate Change stellt in regelmäßigen Abständen Assessment Reports bereit (Houghton et al. 2001); im Rahmen der Biodiversitätskonvention wurde ein Global Biodiversity Outlook erstellt (Secretariat of the CBD 2001); und in Vorbereitung befindet sich ein Millenium Ecosystem Assessment, das die verschiedenen bisherigen assessments integrieren soll (Alcamo et al. 2003). 
richtungen oder Behörden Sammlungen durchzuführen und das gesammelte Material auszuführen, um es im eigenen Land zu analysieren und auszustellen. Die Biodiversitätskonvention hat jedoch die Verfügungsgewalt der Nationalstaaten über die auf dem jeweiligen Territorium befindlichen biologischen Ressourcen völkerrechtlich festgelegt. Im Zuge des Verhandlungsprozesses sind die gerade beschriebenen Praktiken zudem als quasi-koloniale Praktiken kritisiert und delegitimiert worden. Einige Länder haben zudem inzwischen ihre Kontrollen verschärft und zusätzliche Gesetze erlassen. Das wichtigste Beispiel ist hier Brasilien mit dem nationalen Gesetz gegen Biopiraterie. Brasilien wird in vielen Interviews dafür zitiert, dass Forschungsaktivitäten vor Ort erheblich gehemmt werden, weil es schwieriger geworden ist, Forschungsgenehmigungen zu bekommen. In vielen Fällen wird dadurch eine Forschungskooperation mit brasilianischen Stellen erzwungen, wo vorher keine Notwendigkeit zur Kooperation bestand. Zunehmend gilt dies auch für Südafrika und einige andere Schwellenländer, die sowohl über einen großen Artenreichtum innerhalb ihres Territoriums verfügen als auch genügend politisches Gewicht haben, um eigenmächtige Forschungsaktivitäten aus den Wissenschaftszentren zu behindern. So werden Materialien bei der Ein- oder Ausfuhr beschlagnahmt, Forschungsgenehmigungen an die substantielle Beteiligung einheimischer ForscherInnen oder Institute geknüpft, »Biopiratie« wird strafrechtlich verfolgt. Da diese Länder typischerweise auch über eigene Forschungspotentiale verfügen, führen hier Kooperationen häufiger zu Koautorenschaft in MainstreamPublikationen, die dann auch in bibliometrischen Analysen über Datenbanken wie den Science Citation Index sichtbar werden.

Insgesamt hat sich in den vergangenen Jahren also ein erhöhter Druck bemerkbar gemacht, mit anderen zu kooperieren und die national verfügbaren Ressourcen zu bündeln. Kooperationen, u.a. in Form von Koautorenschaft, werden als Notwendigkeit und als quasinatürliche Arbeitsform angesehen, und die hier identifizierten Mechanismen fördern einen selektiven Prozess der Ausweitung der kommunikativen Räume des Wissenschaftssystems über den nationalen Kontext hinweg.

\section{Schlussfolgerungen}

Die hier vorgestellte Untersuchung zur Globalisierung der Wissenschaft hat empirisch gezeigt, dass eine Ausweitung kommunikativer Räume im Sinne grenzüberschreitender Koautorenschaftsmuster keineswegs mit einer Ausdünnung der nationalen Kommunikationsräume einhergeht. Globalisierung bedeutet im Bereich Wissenschaft offenbar nicht, dass Denationalisierung stattfindet. Im Gegenteil haben sich zahlreiche Hinweise auf die bleibende Bedeutung des nationalen Referenzrahmens gefunden. Dies lässt eine Erklärung zu, die davon ausgeht, dass Globalisierung gerade durch die Persistenz nationaler Räume vorangetrieben werden kann. Im Zentrum dieser Argumentation ist die dauerhafte Abhängigkeit der Forschung von nationalen Forschungsfördereinrichtungen, die Anreize zu einer über die Grenzen hinweg schauenden strategischen Suche nach Partnerschaften liefert und darüber hinaus zusätzliche Mittel für die politisch geförderte Verdichtung bestimmter transnationaler Räume bereitstellt. Eine gewisse Ausnahme hiervon bildet die EU-Forschungsförderung, die ja selbst bereits eine transnationale Einrichtung ist. Andererseits wird auch hier die Förderung an die Beteiligung von Forschungseinrichtungen aus möglichst vielen Mitgliedstaaten geknüpft, also eine explizite Verbindung von Forschungsqualität und nationaler Herkunft als Bewilligungskriterium. Schließlich hat der internationale Verhandlungskontext selbst zu einer Stärkung des Prinzips der Nationalstaatlichkeit geführt, da die Legitimität der wissenschaftlichen Assessments an die Beteiligung einer möglichst großen Zahl von Nationen geknüpft ist und die Durchführung von capacity building-Programmen für marginale Entwicklungsländer mit sich bringt. Außerdem hat gerade die Biodiversitätskonvention nationale Verfügungsrechte bereitgestellt, die formale Kooperationen mit denjenigen Ländern 
erst notwendig machen, die über einen besonders hohen Artenreichtum verfügen, der als Forschungsgegenstand weltweites Interesse hervorruft. Dieser Befund steht im Einklang mit den in der Einleitung bereits erwähnten Beiträgen der world polity-Forschung zum Verhältnis von Globalisierung und Nationalstaatlichkeit, in denen gezeigt wird, dass sowohl die zunehmende Verbreitung von nationalstaatlichen Zuständigkeitsbereichen als auch ein steigendes Aktivitätsniveau der Nationalstaaten insgesamt gerade als Ausdruck von Globalisierung zu deuten sind.

\section{Literatur}

Albrow, M. (1996): The global age. State and society beyond modernity, Stanford: Stanford University Press.

Alcamo, J. et al. (2003): Millenium Ecosystem Assessment. Ecosystems and human well-being, Washington et al.: Island Press.

Anheier, H. / Glasius, M. / Kaldor, M. (Hrsg.) (2001): Global Civil Society 2001. Yearbook, Oxford: Oxford University Press.

Basch, L. / Glick Schiller, N. / Szanton Blanc, C. (1994): Nations unbound. Transnational projects, postcolonial predicaments, and deterritorialized nation-states, Amsterdam: Gordon and Breach Science Publishers.

Beaver, D. de B. / Rosen, R. (1978): Studies in scientific collaboration. Part I: The professional origins of scientific co-authorship, in: Scientometrics 1, S.65-84.

Beck, U. (1996): Weltrisikogesellschaft, Weltöffentlichkeit und globale Subpolitik, in: A. Diekmann / C.C. Jaeger (Hrsg.): Umweltsoziologie. Sonderheft 36 der Kölner Zeitschrift für Soziologie und Sozialpsychologie, Opladen: Westdeutscher Verlag, S.119-147.

Beisheim, M. et al. (1999): Im Zeitalter der Globalisierung? Thesen und Daten zur gesellschaftlichen und politischen Denationalisierung, Baden-Baden: Nomos.

Brock, L. (2003): Verlassene Baustellen. Global Governance im Zeichen des Krieges, in: T. Fues, / J. Hippler (Hrsg.): Globale Politik. Entwicklung und Frieden in der Weltgesellschaft, Bonn: Dietz, S.58-89.

Castells, M. (1996) : The rise of the network society. Vol. 1: Information age, Oxford: Blackwell Publishers.

Crawford, E. et al. (Hrsg.) (1993): Denationalizing science, Dordrecht et al.: Kluwer Academic Publishers.

Crouch, C. / Streeck, W. (Hrsg.) (1997): Political economy of modern capitalism. Mapping convergence and divergence, London: Sage.

Dahdouh-Guebas, F. et al. (2003): Neo-colonial science by the most industrialised upon the least developed countries in peer-reviewed publishing, in: Scientometrics 56, S.329-343.

Djelic, M.-L. / Sahlin-Andersson, K. (Hrsg.) (2006): Transnational governance. Institutional dynamics of regulation, Cambridge: Cambridge University Press.

Doremus, P.N. et al. (1998): The myth of the global corporation, Princeton: Princeton University Press.

Drori, G. et al. (2003): Science and the modern world polity. Institutionalization and globalization, Stanford: Stanford University Press.

Dürrschmidt, J. (2002): Globalisierung, Bielefeld: transkript.

Engels, A. (2003): Die geteilte Umwelt. Ungleichheit, Konflikt und ökologische Selbstgefährdung in der Weltgesellschaft, Weilerswist: Velbrück Wissenschaft.

Engels, A. / Ruschenburg, T. / Weingart, P. (2005): Recent internationalization of global environmental change research in Germany and the U.S., in: Scientometrics 62, S.67-85.

Engels, A. / Weingart, P. (1997): Die Politisierung des Klimas. Zur Entstehung von anthropogenem Klimawandel als politischem Handlungsfeld, in: P. Hiller / G. Krücken (Hrsg.): Risiko und Regulierung. Soziologische Beiträge zu Technikkontrolle und präventiver Umweltpolitik, Frankfurt: Suhrkamp, S.90-115. 
Frame, D. J. / Narin, F. / Carpenter, M.P. (1977): The distribution of world science, in: Social Studies of Science 7, S.501-516.

Gaillard, J. / Krishna, V. V. / Waast, R. (Hrsg.) (1997): Scientific communities in the developing world, New Delhi, Thousand Oaks, London: Sage.

Glänzel, W. (2001): National characteristics in international scientific co-authorship relations, in: Scientometrics 51, S.69-115.

Goldman, M. (2004): Imperial science, imperial nature. Environmental knowledge for the World (Bank), in: S. Jasanoff / M.L. Martello (Hrsg.), Earthly politics. Local and global in environmental governance, Cambridge, MA / London: The MIT Press, S.55-80.

Guillén, M. (2001): Is globalization civilizing, destructive or feeble? A critique of five key debates in the social science literature, in: Annual Review of Sociology, S.235-260.

Hall, P. A. / Soskice, D. (Hrsg.) (2001): Varieties of capitalism. The institutional foundations of comparative advantage, Oxford: Oxford University Press.

Houghton, J.T. et al. (Hrsg.) (2001): Climate change 2001. The scientific basis, Cambridge: Cambridge University Press.

Jamison, A. (1982): National components of scientific knowledge, Lund: Research Policy Institute.

Kohler-Koch, B. / Eising, R. (Hrsg.) (1999): The transformation of governance in the European Union. London: Routledge.

Leclerc, M. / Gagné, J. (1994): International scientific cooperation. The continentalization of science, Scientometrics 31, S.261-292.

Levy, D. / Kolk, A. (2002): Strategic responses to global climate change. Conflicting pressures on multinationals in the oil industry, in: Business and Politics 5, S.131-151.

Luhmann, N. (1997): Die Gesellschaft der Gesellschaft, 2 Bd., Frankfurt a.M.: Suhrkamp.

Luukkonen, T. / Persson, O. / Gunnar, S. (1992): Understanding patterns of international scientific collaboration, in: Science, Technology, \& Human Values 17, S.101-126.

Meyer, J.W. (2005): Weltkultur: Wie die westlichen Prinzipien die Welt durchdringen, Frankfurt a.M.: Suhrkamp.

National Science Board (2004): Science and Engineering Indicators 2004, Arlington: National Science Foundation.

Nelson, R. (Hrsg.) (1993): National systems of innovation. A comparative study, Oxford: Oxford University Press.

Ohmae, K. (1995): The end of the nation state. The rise of regional economies, London: Harper Collins.

Paraje, G. / Sadana, R. / Karam, G. (2005): Increasing international gaps in health-related publications, Science 308, 13. Mai, S.959-960.

Pries, L. (1996): Transnationale soziale Räume. Theoretisch-empirische Skizze am Beispiel der Arbeitswanderungen Mexiko - USA, in: Zeitschrift für Soziologie 25, S.456-472.

Raina, D. / Habib, I. (2004): Domesticating modern science. A social history of science and culture in colonial India, New Delhi: Tulika Books.

Reich, R.B. (1993): Die neue Weltwirtschaft. Das Ende der nationalen Ökonomie, Frankfurt/Wien.

Schmidt, V. (2002): The futures of European capitalism, Oxford: Oxford University Press.

Schott, T. (1988): International influence in science. Beyond center and periphery, in: Social Science Research 17, S.219-238.

Schubert, A. / Braun T. (1990): International collaboration in the sciences 1981-1985, Scientometrics 19, S.3-10.

Secretariat of the CBD (2001): Global biodiversity outlook, Montreal: UNEP, WMO, CBD.

Smelser, N.J. (2003): Pressures for continuity in the context of globalization, in: Current Sociology, 51, S.101-112.

Stichweh, R. (2000): Die Weltgesellschaft. Soziologische Analysen, Frankfurt a.M.: Suhrkamp. 
Stichweh, R. (1999): Globalisierung von Wirtschaft und Wissenschaft. Produktion und Transfer wissenschaftlichen Wissens in zwei Funktionssystemen der modernen Gesellschaft, in: Soziale Systeme 5, S.27-41.

Stichweh, R. (1997): Inklusion/Exklusion, funktionale Differenzierung und die Theorie der Weltgesellschaft, in: Soziale Systeme 3, S.123-136.

WBGU (Wissenschaftlicher Beirat Globale Umweltveränderungen) (1996): Welt im Wandel - Herausforderung für die deutsche Wissenschaft, Berlin/Heidelberg: Springer.

Anita Engels, Centrum für Globalisierung und Governance, Institut für Soziologie, Universität Hamburg, Allende-Platz 1, 20146 Hamburg. anita.engels@sozialwiss.uni-hamburg.de

Dipl.-Soz. Tina Ruschenburg, Institut für Weltgesellschaft, Fakultät für Soziologie, Universität Bielefeld, 33501 Bielefeld. tina.ruschenburg@uni-bielefeld.de 


\section{Anhang I - Liste der untersuchten Institute}

\section{USA}

Atlantic Oceanographic and Meteorological Laboratory (AOML), Miami, FL Atmospheric Sciences Competency, NASA Langley Research Center (ATSC/LARC), Hampton, VA Center for Conservation Biology (CCB), Dep. of Biological Sciences, Stanford University, Stanford, CA Climate Monitoring and Diagnostics Laboratory (CMDL), NOAA, Boulder, CO

College of Forest Resources (CFR), University of Washington, Seattle, WA

College of Oceanic Atmospheric Sciences (COAS), Oregan State University, Corvallis, OR

Department of Ecology and Evolutionary Biology (EEBP), Princeton University, Princeton, NJ

Department of Natural Resources (DNR), Cornell University, Ithaca, NY

Department of Oceanography (DO), Texas A\&M University, College Station, TX

Environmental Sciences Division, Oak Ridge National Lab (ESD), Oak Ridge, TN

Energy and Resources Group (ESG), University of California, Berkeley, CA

Environmental Studies Department (ES), University of California, Santa Cruz, CA

Geophysical Fluid Dynamics Laboratory (GFDL), NOAA, Princeton, NJ

Global Environmental Change Program (GECP), Department of Environmental Sciences, University of Virginia, Charlottesville, VA

Goddard Institute for Space Studies (GISS), Columbia University, NY

Goddard Space Flight Center (GSFC), NASA, Greenbelt, MD

Institute of Global Environment and Society (IGES/COLA), Calverton, MD

Lamont-Doherty Earth Observatory (LDEO), Columbia University, Palisades, NY

Lubchenco/Menge Lab (LML), Department of Zoology, Oregon State University, Corvallis, OR

Missouri Botanical Garden (MBG), St. Louis, MO

National Center for Atmospheric Research (NCAR), Boulder, CO

National Geophysical Data Center (NGDC), Boulder, CO

Natural Resource Ecology Laboratory (NREL), Colorado State University, Fort Collins, CO

Naval Postgraduate School (NPS), Monterey, CA

Pacific Marine Environmental Laboratory (PMEL), Seattle, WA

Program for Climate Model Diagnosis and Intercomparison (PCMDI), Lawrence Livermore National Laboratory, Livermore, CA

Program in Atmospheric and Oceanic Sciences (PAOS), University of Colorado, Boulder, CO

Rosenstiel School of Marine and Atmospheric Science (RSMAS), University of Miami, FL

School of Natural Resources and the Environment (SMRE), University of Michigan, Ann Arbor, MI

School of Ocean and Earth Science and Technology (SOEST), University of Hawaii, HI

Scripps Institution of Oceanography (Scripps), University of California, San Diego, CA

Smithsonian Environmental Research Center (SERC), Edgewater, MD

Wisconsin State Herbarium (WSH/DBH), Department of Botany and Herbarium, University of Wisconsin, Madison, WI

Woods Hole Oceanographic Institution (WHOI), Woods Hole, MA

Woods Hole Research Center (WHRC), Woods Hole, MA

World Resources Institute (WRI), Washington, D.C. 


\section{Deutschland}

Alfred-Wegener-Institut für Polar- und Meeresforschung (AWI), Bremerhaven

Biologische Bundesanstalt für Land- und Forstwirtschaft (BBA), Braunschweig

Botanisches Institut und Botanischer Garten (BIBG), Rheinische Friedrich-Wilhelms-Universität, Bonn

Bundesforschungsanstalt für Landwirtschaft (FAL), Braunschweig

Deutsche Sammlung von Mikroorganismen und Zellkulturen (DSMZ), Braunschweig

Deutsches Klimarechenzentrum (DKRZ), Hamburg

Deutsches Primatenzentrum (DPZ), Göttingen

Forschungsinstitut Senckenberg (FIS), Frankfurt a.M.

Fraunhofer-Institut für Atmosphärische Umweltforschung (IFU), Garmisch-Partenkirchen

Institut für Allgemeine Botanik und Botanischer Garten (IABBG), Universität Hamburg

Institut für Küstenforschung (IfK/GKSS), GKSS, Geesthacht

Institut für Ökologie (IÖ), Friedrich-Schiller-Universität, Jena

Institut für Pflanzengenetik und Kulturpflanzenforschung (IPK), Gatersleben

Institut für Zoo- und Wildtierforschung (IZW), Berlin

Max-Planck-Institut für Meteorologie (MPI), Hamburg

Meteorologisches Institut (MIUB), Rheinische Friedrich-Wilhelms-Universität, Bonn

Potsdam Institut für Klimafolgenforschung (PIK), Potsdam

Zoologisches Forschungsinstitut und Museum Alexander König (ZFMK), Bonn 
Anhang II - Datengrundlage für die präsentierten Anteilswerte

Tabelle 7: Anzahl aller Artikel, differenziert nach internationaler, nationaler und ohne externe Koautorenschaft

\begin{tabular}{|c|c|c|c|c|c|c|c|c|c|c|c|}
\hline & 1993 & 1994 & 1995 & 1996 & 1997 & 1998 & 1999 & 2000 & 2001 & 2002 & $\begin{array}{l}1993- \\
2002\end{array}$ \\
\hline \multicolumn{12}{|c|}{ Deutsche Institute } \\
\hline alle Artikel & 591 & 682 & 844 & 875 & 1041 & 1113 & 1113 & 1202 & 1272 & 1354 & 10087 \\
\hline $\begin{array}{l}\text { mit interna- } \\
\text { tionalen } \\
\text { Koautoren }\end{array}$ & 159 & 217 & 289 & 303 & 421 & 449 & 490 & 596 & 620 & 687 & 4231 \\
\hline $\begin{array}{l}\text { nur inländische } \\
\text { Koautoren }\end{array}$ & 129 & 145 & 192 & 183 & 240 & 268 & 265 & 260 & 290 & 317 & 2289 \\
\hline $\begin{array}{l}\text { ohne externe } \\
\text { Koautoren }\end{array}$ & 303 & 320 & 363 & 389 & 380 & 396 & 358 & 346 & 362 & 350 & 3567 \\
\hline \multicolumn{12}{|l|}{ US-Institute } \\
\hline alle Artikel & 3157 & 3407 & 3435 & 3624 & 3522 & 3829 & 3834 & 3979 & 4107 & 4217 & 37111 \\
\hline $\begin{array}{l}\text { mit interna- } \\
\text { tionalen } \\
\text { Koautoren }\end{array}$ & 626 & 756 & 794 & 951 & 1014 & 1241 & 1275 & 1365 & 1489 & 1585 & 11096 \\
\hline $\begin{array}{l}\text { nur inländische } \\
\text { Koautoren }\end{array}$ & 1357 & 1441 & 1477 & 1555 & 1465 & 1667 & 1687 & 1737 & 1800 & 1786 & 15972 \\
\hline $\begin{array}{l}\text { ohne externe } \\
\text { Koautoren }\end{array}$ & 1174 & 1210 & 1164 & 1118 & 1043 & 921 & 872 & 877 & 818 & 846 & 10043 \\
\hline
\end{tabular}

Tabelle 8: Anzahl der internationalen Artikel mit Koautorenadressen in anderen Industrieländern, Entwicklungsländern und Übergangsländern

\begin{tabular}{|c|c|c|c|c|c|c|c|c|c|c|c|}
\hline & 1993 & 1994 & 1995 & 1996 & 1997 & 1998 & 1999 & 2000 & 2001 & 2002 & $\begin{array}{l}1993- \\
2002\end{array}$ \\
\hline \multicolumn{12}{|c|}{ Deutsche Institute } \\
\hline $\begin{array}{l}\text { mit interna- } \\
\text { tionalen } \\
\text { Koautoren }\end{array}$ & 159 & 217 & 289 & 303 & 421 & 449 & 490 & 596 & 620 & 687 & 4231 \\
\hline Industrieländer & 118 & 173 & 223 & 236 & 340 & 352 & 386 & 479 & 498 & 521 & 3326 \\
\hline $\begin{array}{l}\text { Entwicklungs- } \\
\text { länder }\end{array}$ & 27 & 37 & 37 & 44 & 58 & 84 & 62 & 95 & 90 & 122 & 656 \\
\hline Übergangsländer & 25 & 30 & 55 & 60 & 74 & 80 & 89 & 97 & 95 & 129 & 734 \\
\hline \multicolumn{12}{|l|}{ US-Institute } \\
\hline $\begin{array}{l}\text { mit interna- } \\
\text { tionalen } \\
\text { Koautoren }\end{array}$ & 626 & 756 & 794 & 951 & 1014 & 1241 & 1275 & 1365 & 1489 & 1585 & 11096 \\
\hline Industrieländer & 538 & 645 & 685 & 805 & 860 & 1054 & 1064 & 1155 & 1232 & 1296 & 9334 \\
\hline $\begin{array}{l}\text { Entwicklungs- } \\
\text { länder }\end{array}$ & 86 & 125 & 127 & 172 & 176 & 230 & 248 & 266 & 320 & 363 & 2113 \\
\hline Übergangsländer & 40 & 40 & 41 & 52 & 66 & 79 & 90 & 95 & 102 & 99 & 704 \\
\hline
\end{tabular}


Tabelle 9: Anzahl der Artikel mit Koautorenadressen in Entwicklungsländern, differenziert nach Weltregionen

\begin{tabular}{|c|c|c|c|c|c|c|}
\hline & \multicolumn{3}{|c|}{ Deutsche Institute } & \multicolumn{3}{|c|}{ US-Institute } \\
\hline & 1993-1997 & 1998-2002 & 1993-2002 & 1993-1997 & 1998-2002 & 1993-2002 \\
\hline internationale Artikel & 1389 & 2842 & 4231 & 4141 & 6955 & 11096 \\
\hline \multicolumn{7}{|l|}{ Afrika } \\
\hline Östliches Afrika & 9 & 18 & 27 & 24 & 60 & 84 \\
\hline Nördliches Afrika & 7 & 12 & 19 & 7 & 10 & 17 \\
\hline Südliches Afrika & 13 & 33 & 46 & 81 & 102 & 183 \\
\hline Westliches Afrika & 9 & 17 & 26 & 6 & 10 & 16 \\
\hline Zentralafrika & 0 & 4 & 4 & 10 & 6 & 16 \\
\hline \multicolumn{7}{|l|}{ Asien } \\
\hline Ostasien & 35 & 99 & 134 & 185 & 443 & 628 \\
\hline Südzentralasien & 18 & 55 & 73 & 88 & 130 & 218 \\
\hline Südostasien & 18 & 53 & 71 & 33 & 61 & 94 \\
\hline Westasien & 22 & 29 & 51 & 19 & 28 & 47 \\
\hline \multicolumn{7}{|l|}{ Amerika } \\
\hline Mittelamerika & 11 & 12 & 23 & 62 & 202 & 264 \\
\hline Karibik & 6 & 14 & 20 & 6 & 49 & 55 \\
\hline Südamerika & 69 & 151 & 220 & 176 & 379 & 555 \\
\hline \multicolumn{7}{|l|}{ Ozeanien } \\
\hline Melanesien & 1 & 1 & 2 & 24 & 13 & 37 \\
\hline Mikronesien & 1 & 0 & 1 & 10 & 7 & 17 \\
\hline Polynesien & 0 & 0 & $\mathbf{0}$ & 3 & 3 & 6 \\
\hline
\end{tabular}

